\title{
The Development of Diamond Strength Testing System based on Microprocessor
}

\author{
Kongjun Bao ${ }^{1, \mathrm{a}}$, Rui $\mathrm{Cao}^{1, \mathrm{~b}}$ and Yinhua $\mathrm{Li}^{2, \mathrm{c}}$ \\ ${ }^{1}$ School of Computer and Communication Engineering \\ ${ }^{2}$ School of Electrical and Information Engineering \\ ${ }^{1,2}$ Zhengzhou University of Light Industry, Zhengzhou, 450002, China \\ abaokongjun@qq.com, ${ }^{b}$ caoruii@126.com, ${ }^{c}$ zzfcc@126.com
}

\begin{abstract}
In order to improve the quality of diamond and other super-hard materials, fast and accurate detection of its strength is significant. The System use air pressure, broken automatic detection, automatic samples, testing is completed automatically returned. By resistance strain gauges to the pressure value is converted to voltage signal, after a highprecision A / D converter chip into a numerical quantity, given to high-performance MCU processing. Sample test system can be set according to sample size, data communications, data statistics and report printing, with a large-screen LCD display, dynamic display of strength parameters of the distribution curve. System is reliable test data stability, good repeatability and high reliability.
\end{abstract}

Keywords: Strength testing, Hardness testing, Testing Equipment, Smart Meter, Microprocessor

\section{Introduction}

Super-hard materials products are essential tools for ultra-precision machining. Represented by the diamond super-hard materials have been widely used in building materials, machinery, electronics, petroleum, geology, automobile, electrical, aerospace and other industries and large-scale integrated circuit chips, semiconductor wafers coated, high wear high-tip technology aircraft tires areas. China's diamond sorting and testing standards with foreign standards can't be compared with the device also has fallen behind, especially the lack of precision testing equipment and the error range of the larger issues. Analysis of existing reason, the key is to improve the detection system performance of the automation and sensors, to improve the accuracy and sensitivity. China's diamond industry is focused on the production of high-grade synthetic diamond, the development parameters of a high standard of diamond testing equipment is imminent [1].

\section{Research of Diamond Detection System}

\subsection{Common Test Methods of Diamond}

Stress strength parameters of the diamond measured points the way to load, there are two: First, the facilities contained in the monomer (particles), the second is applied are set out in groups (particle size). The nature of the load, there are two points: First, static load, the second is dynamic load. Determination results of these methods, there are several indicators 
of the current value to define, but in essence, the ultimate strength by three indicators: single static strength, dynamic load impact strength of single, group dynamic load Impact strength.

\subsection{Problems in Existing Equipment}

China's diamond single particle compression test equipment is not high degree of automation common, time-consuming and laborious testing work; measurement method is simple, small measurement range, measurement accuracy is low, and the disadvantage of high prices, can't meet industry needs [2].

\subsection{Significance of Research Projects}

It will be implemented in the past, static strength test of artificial computer-controlled to automatically detect, record, calculate, analyze, print, eliminate the external factors on the detection quality in the use of static pressure suffered in their environment, state of shock and thermal shock, making test results more scientific, impartial, international standardization. Improve the stability of diamond grades for diamond products expand the international market and enhance the international competitiveness of the foundation. Super-hard materials for China to further enhance the level of technology to achieve a variety of synthetic processes, improve product quality, modern management and the promotion of synthesis of super-hard materials and technology, there are certain references.

\section{Technical Analysis of System Design}

\subsection{Technical Requirements for Strength Tester}

- design a diamond in accordance with national standard single-particle strength testing program;

- Automatic pressure with the motor, eliminating that error: Maximum protection, $100 \mathrm{~kg}$ return; empty process automatically returns; automatic data processing; good accuracy, good repeatability;

- Automatic detection of pressure, maximum pressure range of $0 \sim 200 \mathrm{~kg}$; measurement accuracy of $1 \mathrm{~g}$;

- Can automatically display the levels of parameters;

- The Function for various parameter settings

\subsection{The Question must be considered in the Design of the System}

- Based on the above design requirements, the design compressive strength tester diamond single particle to be considered when the following six aspects:

- Select the appropriate pressure sensor chip, depending on the actual needs and the kinds of pressure sensors to determine the performance parameters;

- Choose the right SCM;

- Select the appropriate operational amplifier;

- Select the appropriate A / D conversion device;

- Design of microcontroller and A / D converter interface circuit; 
- Well-designed man-machine interface.

\subsection{The System Design Analysis}

- The pressure sensor design requirements to consider the maximum pressure range 0 $100 \mathrm{~kg}$, the sensor's measurement range greater than $100 \mathrm{~kg}$, so the choice of the resistance strain gauge design.

- The system must have automatic data processing, accurate and repeatable. A complex data operations; need microcontroller has enough memory and high speed, and development costs to the end [3].

- To meet the design requirements, the sensor amplifier circuit is critical, it is related to the data collected by the system are correct. The integrated operational amplifier on the linearity requirements of high and requires a differential amplifier. Design requirements for measurement accuracy of $1 \mathrm{~g}$, so the required number of bits in $\mathrm{AD} 17$ or more at least, but does not require the speed of conversion. In order to ensure the accuracy of the design in the $\mathrm{AD}$ conversion to keep a certain space median to prepare for future system upgrades.

- Good man-machine interface, operation in favor of workers. Used in the design as much as possible to reduce the number of keys; only three control buttons, to avoid complicated operations, on the display with LCD display, friendly interface.

\subsection{The Overall Program Design}

Figure 1 shows: a small test measured the solid particles, such as the artificial Diamond, gems, etc., all-speed motor in the pressure, through the pressure value of the resistance strain gauge is converted to voltage amplifier and $\mathrm{AD}$ after delivery to the microcontroller through microcontroller to detect small particles of each solid exposure to the maximum pressure value, while the microcontroller controls the motor to stop the motor pressure, and automatically return. After 40 solid when measuring small particles, by the microcontroller through the LCD to normal by the static pressure in the form of small particles of solid section shows the number, while small particles of solid shows the measured maximum pressure to bear by the average, the number of particles greater than the average, less than the average particle number and less than $1 / 2$ the average number of particles. When the pressure exceeds $100 \mathrm{~kg}$, or a blank stroke, the motor is automatically returned [4].

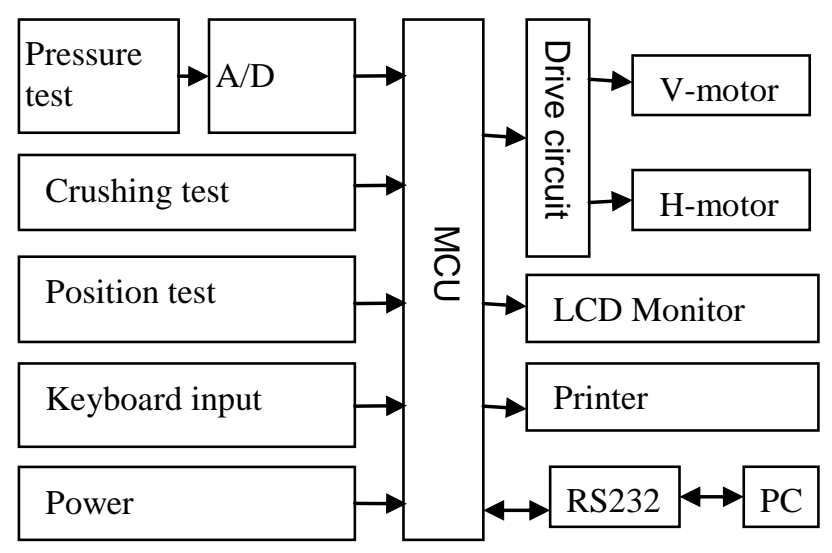

Figure 1. Overall System Block Diagram 


\section{Hardware System Design}

Compressive strength of diamond tester system used in the main components: ATMEGA16, 20 bits A / D device CS5513, differential operational amplifier OP2277, LCD module LCM12864, strain gauge sensors.

\subsection{Main Function Devices}

Single chip is still the core components of the system, the choice of Atmel's microcontroller chip ATMEGA16, shown in Figure 2. It's cost effective, built-in rich, cheap, fair enough supply. And it is more soft support for free development, the development of low cost; it can meet the design, acquisition, control and data processing needs.

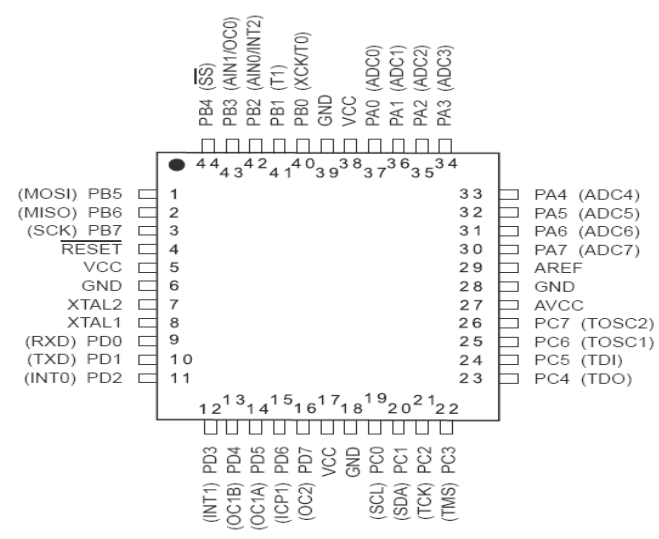

Figure 2. ATMEGA16 Pin

\subsection{The Sensor Amplifier Circuit}

The role of the sensor amplifier is to amplify the input signal to the ADC within the acceptable range. Generally use the pre-amplifier integrated operational amplifier, should be used to select according to the actual needs of the integrated operational amplifier. Selected based on its performance parameters: differential-mode input resistance, output resistance, input offset resistance, current, and temperature drift, open-loop differential gain, common mode rejection ratio and the maximum voltage amplitude and so on.

Sensor amplifier circuit shown in Figure 3, the strain sensor output voltage is very weak, and subject to the amplified sample can be sent to AD, in which R1, R2, C1, C2, C3 together to form a low pass filter.

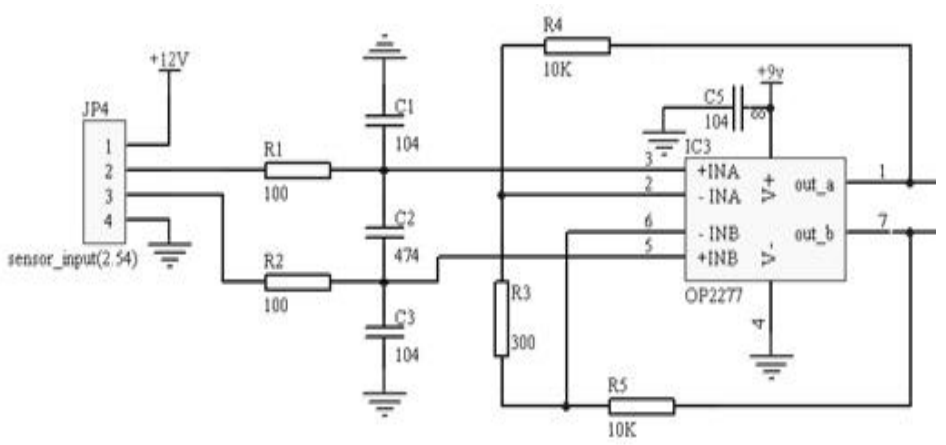

Figure 3. Sensor Amplifier Circuit 


\subsection{A / D Sampling Circuit}

CRYSTAL CS5513 is produced by the United States 20bit serial output A / D converter.The 20 bit A/D device CS5513. In the A / D bits in full compliance with the requirements of this design, the speed can be competent and cheap, helps reduce system cost [5].

A/D sampling circuit shown in Figure 4. Data lines connected ATMEGA16 microcontroller SD0 PD3 pin. MCU clock input termination PD4. CS5513 also use the data and clock terminal pin leads for easy connection microcontroller interrupt pin.

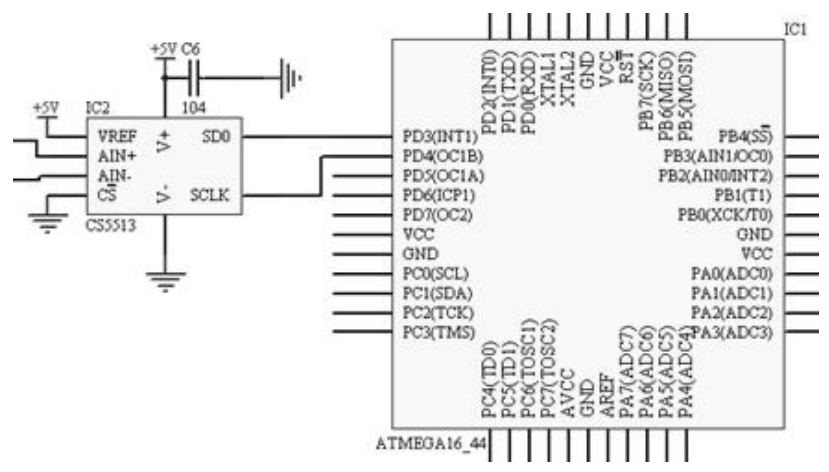

Figure 4. A / D Sampling Circuit

\subsection{Liquid Crystal Display Circuit}

LCD module design uses FYD12864-0402B. YD12864-0402B is a kind of 4 / 8 bit parallel, 2-wire or 3-wire serial interface to a variety of ways, includes the national standard first-line, second point Simplified Chinese font Graphic LCD module-shaped array.

LCD display circuit shown in Figure 5, 1 and 2 pins for the LCD module's ground and power, 19 and 20 pins for the LCD backlight power supply and ground.

To improve detection accuracy and avoid the pressure head and the pad hardness, surface roughness effects on the compressive strength test, by increasing the sample size is the practical approach. This design uses the sample size was 80 , when the test finished 80 of the maximum static pressure value, these particles through the microcontroller to calculate the normal distribution, through the LCD display out.

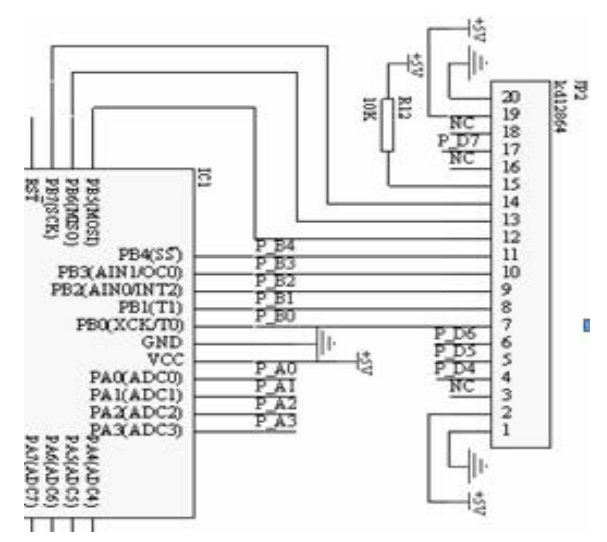

Figure 5. Liquid Crystal Display Circuit 


\subsection{Communication Interface Circuit Design}

Using RS232 serial interface circuit to Asynchronous communication .Because RS232 logic electronic ground is symmetrical, with TTL, CMOS logic levels are completely different. Logic "1" level is between $-5 \sim-15 \mathrm{~V}$, the logic level " 0 " between $+5 \sim+15 \mathrm{~V}$, the noise tolerance of $2 \mathrm{~V}$, which requires the receiver to recognize the signal as low as $+3 \mathrm{~V}$ logic "0", the high-3V signal to a logic "1", apparently, to communicate between the two signal levels must be converted. Therefore, the system uses a wide voltage level converter chip MAX232, MAX232 is a single-supply dual RS-232 transmit / receive chip, using a single +5 $\mathrm{V}$ power supply, it can constitute a standard RS-232 communication interface, conversion circuit shown in Figure 6.

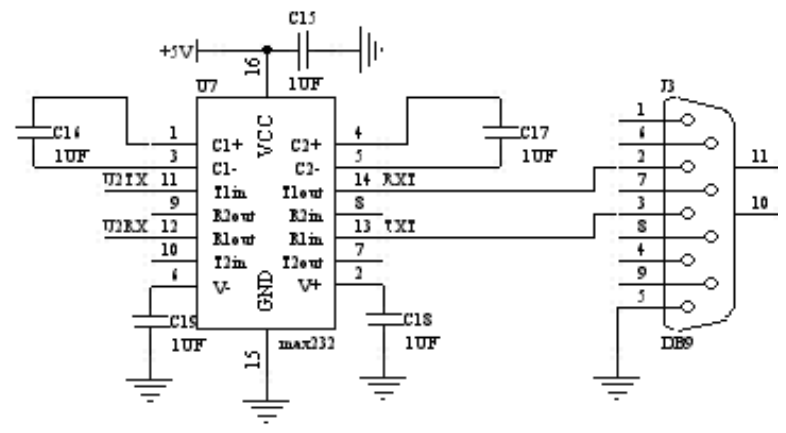

Figure 6. RS-232 Communication Interface Circuit

\subsection{The Data Processing Method}

This design is based on ATMEGA16 microcontroller and digital signal processing algorithms to solve interference problems rise diamond static strength during the measurement with pulsating bring. In the trial focused on avoiding the pressure head, pad hardness and surface roughness on the compressive strength testing. According to experience, the compressive strength of synthetic diamond typically follow a normal distribution, so most of the time we follow the normal distribution to handle test data [6]. This is undoubtedly an approximate approach. In fact, sometimes some of the compressive strength of the diamond does not follow a normal distribution. Assuming, a group of diamond overall mean $\mu$ (unknown), the overall standard deviation $\sigma$ (unknown), the sample mean of the sample, the sample standard deviation of $\mathrm{S}$, the sample size is $\mathrm{n}$. According to the probability of statistical knowledge,

$$
\frac{\bar{X}-\mu}{S / \sqrt{n}} ;
$$

Obey t distribution. That

$$
\frac{\bar{X}-\mu}{S / \sqrt{n}} \sim \mathrm{t}(\mathrm{n}-1) ;
$$

Wherein, $t(n-1)$ does not depend on the distribution of any of the parameters. It can calculate the mean $\mu$ overall confidence level of $95 \%(1-\alpha=95 \%)$ confidence interval:

$$
\left(\bar{X} \pm \frac{S}{\sqrt{n}} t_{w} / 2\right)(n-1)
$$


In other words, based on detection results of the samples taken on the calculated overall average compressive strength of these diamond $\mu$ falls $95 \%$ confidence interval

$$
\left(\left(\bar{X}+\frac{S}{\sqrt{n}} t_{w / 2}\right)(n-1),\left(\bar{X}+\frac{S}{\sqrt{n}} t_{w / 2}\right)(n-1)\right) ;
$$

The $\frac{t \alpha}{2(n-1)}$ can be found from the $\mathrm{t}$ distribution table. For the smaller sample size $\mathrm{n}, \mathrm{t}$ distribution and the standard normal distribution were quite different. But when $\mathrm{n}$ is large enough, $\mathrm{t}$ distribution similar to the standard normal distribution.

$$
\frac{t \alpha}{2(n-1)} \approx \frac{z \alpha}{2}
$$

In the past, due to the compressive strength testing equipment is relatively backward, diamond compressive strength testing time-consuming work. Now, with the use of a pressure sensor to detect the intensity measurement instruments and data processing software applications, detection and calculation of the compressive strength of diamond has become easy. Therefore, increasing the sample size in the diamond compressive strength testing work, to improve the detection accuracy of the method is feasible [7]. This helps to reduce the controversy in synthetic diamond production, sale and use of the identification results due to the emergence of different grades of existence, avoiding the head and under the conditions of pressure pad hardness, surface roughness on the compressive strength testing, testing the accuracy of the results increases as the sample size increases. When the sample size increased to 80 , the overall mean $\mu$ small fluctuations calculated based on test results of diamond compressive strength values. Description detected either by the different units at different times. the possibility of inconsistent results obtained becomes small. Therefore, the sample size used in this design is 80 , when 40 after detecting the maximum static duty, these particles through a normal single-chip meter, the LCD shows it.

\section{Design of the System Software}

Software design includes not only the main program, but also including liquid crystal display subroutine, subroutines key judgments, AD sampling subroutine, subroutines to determine particle pressure values, compared particle pressure conversion subroutine, subroutines, and other motor program module returns [8].

Main processes, as shown in Figure 7. Main program is initialized, start the motor, start measuring the pressure and make diamond A / D sampling. Comparison of the pressure conversion and then call the subroutine, if the maximum pressure on the diamond, turn off the motor and began to return. Did not reach the maximum continued A / D sampling, until it reaches maximum pressure. After returning the mot or to determine whether measurement of the number of diamond particles to 80 , if the pressure reaches 80 the average, less than the average number, the number is greater than the average, less than half the average The calculated and displayed through the LCD. If the number of diamond particles smaller than 80 , the particle count plus 1, through the LCD shows the number of particles measured. End of diamond grains in the test, if the control buttons NEXT to 0 for the next particle measurements, record data or LCD display. In the NEXT even after the motor is 0 , return the judge to decide whether to start the next round of measurement. 


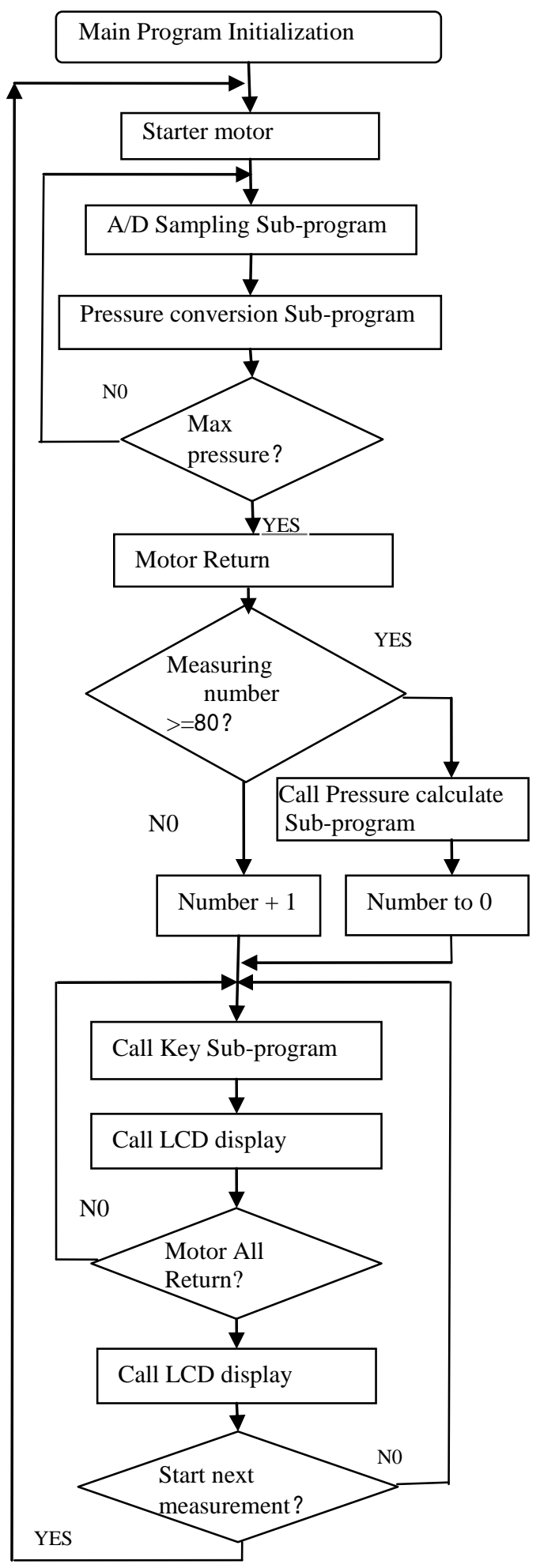

Figure 7. The Main Program Flow Chart 


\section{Analysis of Experimental Data}

First, the next with the same sanding pressure head, and turn on the pressure head has been polished with a diamond-treated 1800, No. (1) pressure head, 720 has been polished diamond indenter No.(2), and polishing pressure head No. (3), Samples were tested on the MBD 45/50 to 80 diamond value of the average single particle strength as the test results. Then, the pressure head on the number (1) and polishing head again under the pressure of the sample testing. Test results are shown in Table 1.

Table 1. Test Result

Unit: $\mathbf{N}$

\begin{tabular}{|c|c|c|c|c|c|c|c|}
\hline $\begin{array}{c}\text { Sample } \\
\text { Type }\end{array}$ & $\mathbf{A}$ & B & $\begin{array}{c}\text { Testing } \\
\text { times }\end{array}$ & $\mathbf{X}$ & M & $X$ & $M$ \\
\hline \multirow{5}{*}{ MBD } & \multirow{8}{*}{ Brushed } & (1) & 1 & 172 & 154 & \multirow{10}{*}{187} & \multirow{10}{*}{160} \\
\hline & & & 2 & 176 & 163 & & \\
\hline & & & 3 & 174 & 148 & & \\
\hline & & (2) & 1 & 183 & 158 & & \\
\hline & & & 2 & 184 & 144 & & \\
\hline \multirow{5}{*}{$\begin{array}{c}45 \\
/ \\
50\end{array}$} & & & 3 & 165 & 155 & & \\
\hline & & (3) & 1 & 248 & 176 & & \\
\hline & & & 2 & 205 & 179 & & \\
\hline & Polished & (1) & 1 & 183 & 164 & & \\
\hline & & & 2 & 184 & 156 & & \\
\hline
\end{tabular}

Table 1 shows, (1) (2) (3) the pressure head Roughness becomes smaller, so the test results become bigger. Different roughness of the pressure head testing, the number of $\mathrm{M}$ characterized by the compressive strength of all the degree of dispersion characterization than the average compressive strength of $\mathrm{X}$ is much smaller degree of dispersion, so to ensure that test results from the stability point of view, with the public Characterization of a more reasonable number of compressive strength.

\section{References}

[1] W. M. Johnson and A. J. Rapoff, "Microindentation in bone: Hardness variation with five independent variables”, Journal of Materials Science: Materials in Medicine, dio: 10.1007/s10856-007-2306-4, vol. 8, no. 4, pp. 591-597.

[2] C. Fares, T. Boukharouba, M. El Amine Belouchrani and A. Britah, "Determination of the Hardness of the Oxide Layers of 2017A Alloys", Damage and Fracture Mechanics, dio: 10.1007/978-90-481-2669-9_1, (2009), pp. 1-10.

[3] N. A. Khilo, P. I. Ropot, N. S. Kazak, V. N. Belyi and A. G. Mashchenko, "Optical method for determining the hardness of metals and alloys", Journal of Applied Spectroscopy, doi: 10.1007/s10812-009-9177-z, vol. 76, no. 3, pp. 434-439.

[4] Y. Li, H. Road and Z. Yan Zhong, "Computer Vision Inspection of the diamond net Application", Diamond \& Abrasives Engineering, vol. 169, (2009) February, pp. 69-70.

[5] C. Zeng Shen, J. Zheng, W. Con xian and W. Hai yong, "An Investigation on Force Controlling Method for Measuring Fracture Strength and Toughness of Diamond Film", Journal of Experimental Mechanics, (2001) February, pp. 14.

[6] K. O. Mikaelian, (Lawrence Livermore National Laboratory, Livermore, "Testing diamond strength at high pressure", CA 94551, United States), Source: Diamond and Related Materials, vol. 20, no. 10, (2011) November, pp. 1340-1343.

[7] O., Y., S., R. and I., "Tensile test of diamond-like carbon thin films by nanomaterials testing system", Source: Nippon Kinzoku Gakkaishi/Journal of the Japan Institute of Metals, vol. 76, no. 5, (2012) May, pp. 327-331.

[8] R. Morrell, R. Danzer and P. Supancic, "Meso-scale mechanical testing methods for diamond composite materials", Source: International Journal of Refractory Metals and Hard Materials, vol. 28, no. 4, (2010) July, pp. 508-515. 


\section{Authors}

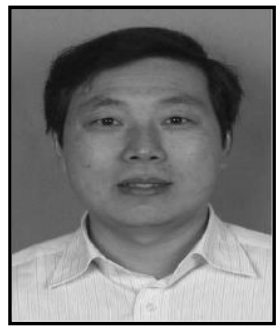

Kongjun Bao received his BS in Electrical Technology from Zhengzhou University of Light Industry, Zhengzhou, China, in 1987. He got his MS in Computer Application from Huazhong University of Science and Technology, Wuhan, China, in 2003. He is an Associate Professor in the School of Computer and Communication at Zhengzhou University of Light Industry. His research interests include network database and multimedia.

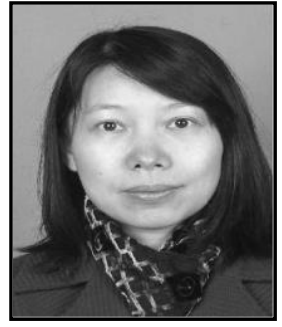

Rui Cao received her BS in Electrical Technology from Beijing Institute of Technology, Beijing, China, in 1989. She got her MS in Communication Engineering from National University of Defense Technology, Changsha, China, in 2002. She is an Associate Professor in the School of Computer and Communication at Zhengzhou University of Light Industry. Her research interests include signal processing and the embedded system.

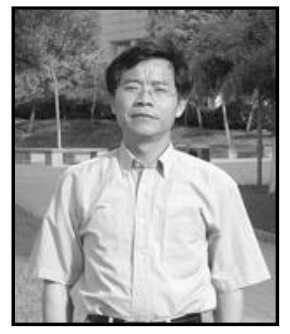

Linhua Li received his BS in Electrical Technology from Zhengzhou University of Light Industry, Zhengzhou, China, in 1987. He got his MS in Electrical Technology from Southeast University, Nanjing, China, in 1993. He is a Professor in the School of Electrical and Information Engineering at Zhengzhou University of Light Industry. His research interests include Smart meters, intelligent sensor technology, Embedded Application Systems. 